\title{
Channel Access and Power Control for Mobile Crowdsourcing in Device-to-Device Underlaid Cellular Networks
}

\author{
Yue Ma $\mathbb{D}^{1},{ }^{1}$ Li Zhou $\mathbb{D}^{D},{ }^{2}$ Zhenghua Gu, ${ }^{1}$ Yang Song, ${ }^{1}$ and Bin Wang ${ }^{1}$ \\ ${ }^{1}$ China Aerodynamics Research and Development Center, Mianyang 621000, China \\ ${ }^{2}$ School of Electronic Science, National University of Defense Technology, Changsha 410073, China
}

Correspondence should be addressed to Li Zhou; zhouli2035@nudt.edu.cn

Received 2 September 2017; Accepted 4 December 2017; Published 4 January 2018

Academic Editor: Kun Bai

Copyright (C) 2018 Yue Ma et al. This is an open access article distributed under the Creative Commons Attribution License, which permits unrestricted use, distribution, and reproduction in any medium, provided the original work is properly cited.

With the access of a myriad of smart handheld devices in cellular networks, mobile crowdsourcing becomes increasingly popular, which can leverage omnipresent mobile devices to promote the complicated crowdsourcing tasks. Device-to-device (D2D) communication is highly desired in mobile crowdsourcing when cellular communications are costly. The D2D cellular network is more preferable for mobile crowdsourcing than conventional cellular network. Therefore, this paper addresses the channel access and power control problem in the D2D underlaid cellular networks. We propose a novel semidistributed network-assisted power and a channel access control scheme for D2D user equipment (DUE) pieces. It can control the interference from DUE pieces to the cellular user accurately and has low information feedback overhead. For the proposed scheme, the stochastic geometry tool is employed and analytic expressions are derived for the coverage probabilities of both the cellular link and D2D links. We analyze the impact of key system parameters on the proposed scheme. The Pareto optimal access threshold maximizing the total area spectral efficiency is obtained. Unlike the existing works, the performances of the cellular link and D2D links are both considered. Simulation results show that the proposed method can improve the total area spectral efficiency significantly compared to existing schemes.

\section{Introduction}

With the development of intelligent handheld devices, mobile crowdsourcing is emerging as an efficient strategy to improve the user experiences of applications in wireless networks [1]. With the help of ubiquitous mobile users who can share the information between each other, the large-scale crowdsourcing tasks can be facilitated [2]. The D2D communications can realize the high-speed data transmission among mobile users with lower power and shorter delay, which largely eases collaborations among participants [3-6]. Therefore, the D2D cellular network is more preferable for mobile crowdsourcing than conventional cellular network [2].

Despite making full use of radio resources, the underlaid scenario leads to the coexistence of cellular and D2D communications in the same frequency band, which brings cross-tier and cotier interferences. Therefore, interference management becomes essential in the underlaid scenario.
Transmit power control and channel allocation are widely used to mitigate interference in wireless networks. Different from ad hoc networks and traditional D2D technologies (e.g., WiFi direct), the base station (BS) can assist the DUE pieces in controlling transmit power and allocate the subchannels in the underlaid D2D cellular networks [7]. Compared to DUE pieces, the BS has a more powerful signal processing ability and more easily obtains useful information for resource allocation. Generally, the resource management methods in D2D underlaid cellular networks can be classified into two main categories: the network-controlled device-assisted method and the network-assisted device-decided method [814]. In the network-controlled device-assisted method, each DUE needs to report its own information (e.g., channel state information, CSI) to the BS, and then the BS allocates the radio resource to each DUE [8-10]. In [8], a joint power control and mode selection strategy was proposed to improve the overall system performance. To protect existing cellular 
links, the authors in [9] proposed a dynamic power control method to limit the D2D transmission power. In [10], the centralized algorithm was used to control the power of both the cellular user equipment (CUE) and DUE pieces. Note that the network-controlled device-assisted method requires global information at the BS. However, when the density of DUE pieces is high, the overhead to transmit global information to the BS will reduce the system efficiency significantly and it may be unacceptable for real-time processing.

For the network-assisted device-decided method, each DUE selects suitable channels and decides the transmission power according to a minimum transmit power criterion and the power control instruction from the BS [11]. In this scheme, the BS does not need to know global information about each DUE and the overhead can be reduced significantly. In [11], the authors proposed a network-assisted devicedecided scheme to jointly select the channels and adjust the transmission power for DUE pieces. However, the number of active DUE pieces on one certain channel is not limited, which may lead to severe interference among the DUE pieces. In [10], based on the statistical features of CSI, distributed on-off power control algorithm was proposed and the fixed threshold was applied to control the number of active DUE pieces. The fixed threshold in [10] is the same for all DUE pieces. In [12], to minimize the D2D transmit power, a statistical features-based power control with opportunistic access control was proposed. Unlike [10], each DUE has its own optimal access threshold in [12].

However, most existing channel access schemes only consider the performance of DUE pieces, while the performance of the cellular user is ignored [10-12]. Moreover, although the works in $[10,12]$ control the power of DUE pieces according to a minimum transmit power criterion, the interference from DUE pieces to the cellular user is not controlled accurately. In this paper, we focus on the power and channel access control problem in a network-assisted device-decided scheme. The main contributions of this paper are as follows:

(1) A novel network-assisted power and channel access control scheme is presented. The proposed scheme can control the interference from DUE pieces to the cellular user accurately. Moreover, the proposed scheme is semidistributed, which can guarantee the performances of both the cellular link and D2D links without high information feedback overhead.

(2) For the proposed method, we apply the stochastic geometry tool and derive analytic expressions including the coverage probabilities of both the cellular link and D2D links.

(3) The Pareto optimal access threshold maximizing the total area spectral efficiency is obtained. Different from the existing works, the performances of the cellular link and D2D links are both considered in obtaining the optimal access threshold.

In the D2D cellular network, both the cellular users and D2D users may participate in the crowdsourcing tasks. The proposed scheme considers the performances of both the cellular link and D2D links, which can allow cellular users

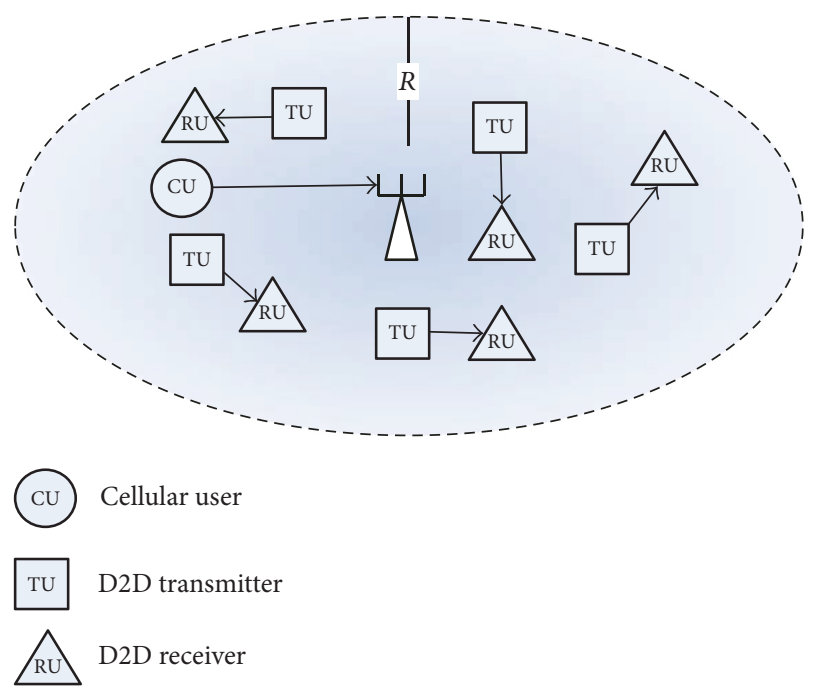

Figure 1: Illustration of a D2D underlaid cellular network.

and D2D users to better carry out crowdsourcing tasks. On the other hand, large-scale crowdsourcing tasks need the participation of ubiquitous mobile users, which is based on the network with high performance. The proposed scheme can improve the system efficiency significantly without high information feedback overhead, which is preferable for largescale crowdsourcing tasks.

\section{System Model}

The architecture of a D2D underlaid network is considered, as shown in Figure 1. In this model, we denote the circular disk $C$ with radius $R$ as the coverage region of a base station (BS) centered at the origin. There are one BS (i.e., $k_{0}$ ) and $N_{d}^{\text {tol }}$ D2D pairs. We assume that the cell has one cellular user equipment (CUE) piece on one subchannel. Denote the CUE as $s_{0}$. Each D2D pair comprises one transmit DUE (TU) piece and one DUE reception (RU) piece. Denote TU and RU pieces as $\left(u_{t_{1}}, u_{t_{2}}, \ldots, u_{t_{d}^{\text {tol }}}\right)$ and $\left(u_{r_{1}}, u_{r_{2}}, \ldots, u_{r_{N_{d}^{\text {tol }}}}\right)$. The DUE pieces can share the uplink subchannel of the CUE $s_{0}$. All nodes are equipped with one antenna. The CUE is uniformly located in the region $C$. The locations of the TU pieces follow the independent homogeneous Poisson point processes (PPPs) $\Phi_{\mathrm{DU}}$ with density $\lambda_{\mathrm{DU}}$. The intended RU pieces are uniformly and independently located within the distance $R_{d}$ of their associated TU pieces. Under the given assumptions, $\mathbb{E}\left[N_{d}^{\mathrm{tol}}\right]=\lambda_{\mathrm{DU}} \pi R^{2}$. The CUE operates at a fixed transmission power, defined as $P_{s_{0}}$.

\section{Network-Assisted Channel Access and Power Control for DUE Pieces}

3.1. Semidistributed Channel Access. In this subsection, we present a semidistributed channel access method, which is an effective interference mitigation method that requires assisted information from the BS. In Figure 2, the subchannel represents a particular time slot. For example, at slot 1, the 


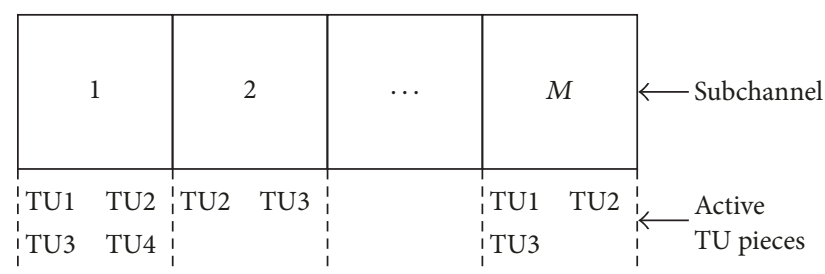

FIGURE 2: The illustration of D2D user's channel access.

active TU1, TU2, TU3, and TU4 use the same frequency band. As in [10-12], the link-gain is applied as the access metric. We use a nonnegative threshold to limit the number of DUE pieces on the subchannel and reduce the interference to cellular users. Moreover, each D2D pair has its own threshold, which is calculated by itself based on the statistical parameters from the BS. The access threshold is calculated in TU. The RU can obtain the link-gain by channel estimation and feed back it to the TU. $G_{u_{n}, i}$ denotes the threshold for the D2D pair $u_{n}$ (i.e., $\left.\left(u_{t_{n}}, u_{r_{n}}\right)\right)$ on the subchannel $i$. Then, in order to avoid the overheads for reporting global information to the BS, each D2D pair decides whether to access the current subchannel solely based on its own link-gain and the threshold independently. Specifically, when the link-gain of the D2D pair $u_{n}$ on subchannel $i$ is larger than $G_{u_{n}, i}$, the D2D pair $u_{n}$ is active on the subchannel $i$; otherwise, the $\mathrm{D} 2 \mathrm{D}$ pair $u_{n}$ is idle on the subchannel $i$.

The transmit probability of the D2D pair $u_{n}$ in the subchannel $i$ is given by

$$
\begin{aligned}
P S_{u_{n}, i} & =\mathbb{P}\left[\left|h_{u_{t_{n}}, u_{r_{n}}, i}\right|^{2} d_{u_{t_{n}}, u_{r_{n}}}^{-\alpha}>G_{u_{n}, i}\right] \\
& =\exp \left(-G_{u_{n}, i} d_{u_{t_{n}}, u_{r_{n}}}^{\alpha}\right),
\end{aligned}
$$

where $h_{u_{t_{n}}, u_{r_{n}}, i}$ and $d_{u_{t_{n}}, u_{r_{n}}}$ represent the small-scale fast fading and distance from TU $u_{t_{n}}$ to RU $u_{r_{n}}$ on subchannel $i$, respectively. $\alpha$ is the path-loss exponent. The Rayleigh distribution is assumed (i.e., $h_{u_{t_{n}}, u_{r_{n}}, i} \sim \mathscr{C} \mathscr{N}(0,1)$ ). The proposed algorithm is semidistributed as each $\mathrm{D} 2 \mathrm{D}$ pair decides whether to access the subchannel by its own channel gain $\left|h_{u_{t_{n}}, u_{r_{n}}, i}\right|^{2}$ and network-assisted threshold $G_{u_{n}, i}$. Note that the threshold $G_{u_{n}, i}$ not only has an influence on the performance of cellular users, but also determines the performance of the D2D links. On the one hand, larger $G_{u_{n}, i}$ reduce both the interference from DUE pieces to cellular users and the inter-D2D interference. On the other hand, larger $G_{u_{n}, i}$ leads to smaller number of active DUE pieces. Therefore, in order to balance these two competing factors, $G_{u_{n}, i}$ is optimized in Section 5.

3.2. Semidistributed Power Control. Based on the channel access method in Section 3.1, the semidistributed power control strategy is presented as shown in Figure 3.

The core idea of the proposed power control strategy mainly includes the following.

Step 1. The BS broadcasts the statistical parameters for each D2D pair to calculate the threshold $G_{u_{n}, i}$.

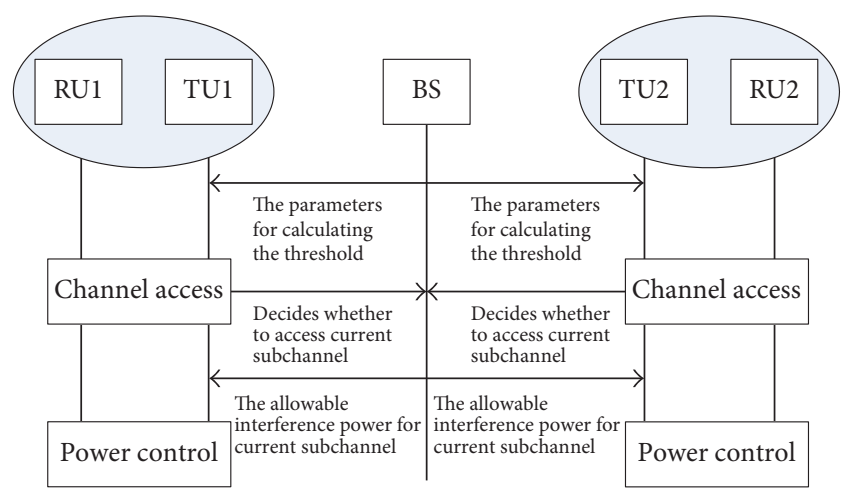

FIgURE 3: Network-assisted, device-decided channel access and power control scheme.

Step 2. Each D2D pair decides whether to access the current subchannel by its own channel gain $\left|h_{u_{t_{n}}, u_{r_{n}}, i}\right|^{2}$ and threshold $G_{u_{n}, i}$ and then transmits the result to the BS.

Step 3. The BS calculates the allowable power for each TU according to both the CUE performance requirement and the number of active D2D pairs on the current subchannel and then transmits the allowable power to each TU.

Step 4. According to the assistant power instruction broadcasted from the BS and the maximum transmission power for the TU, each TU autonomously adjusts the transmit power independently.

Steps 1 and 2 refer to Section 3.1. In the following, without loss of generality, we take the subchannel $i$ as an example and the index of subchannel $i$ will be ignored. For example, $h_{u_{t_{n}}, u_{r_{n}}, i}$ will be replaced by $h_{u_{t_{n}}, u_{r_{n}}}$. The detailed processes of Steps 3 and 4 will be presented. The conclusions can be easily extended to other subchannels.

In order to ensure the link reliability of the CUE, the proposed power control aims at adjusting the transmission power of TU pieces. Denote the target $I_{k_{0}}$ as the allowable interference power from $\mathrm{TU}$ pieces to the CUE on the subchannel $i$. According to Steps 1 and 2 of the proposed power control algorithm, the BS can count the number of active TU pieces that prefer the subchannel $i$ to perform the $\mathrm{D} 2 \mathrm{D}$ communications. Hence, the allowable transmission power unit for $\mathrm{TU} u_{t_{n}}$ is given by

$$
P L_{u_{t_{n}}}=\frac{I_{k_{0}}}{N_{d}\left(\left|h_{u_{t_{n}}, k_{0}}\right|^{2} d_{u_{t_{n}}, k_{0}}^{-\alpha}\right)},
$$

where $N_{d}$ is the number of active D2D pairs on the subchannel $i$, and $\mathbb{E}\left[N_{d}\right]=\lambda_{\mathrm{DU}} P S_{u_{n}} \pi R^{2} . P S_{u_{t_{n}}}$ is the access probability for D2D links on the subchannel $i . h_{u_{n}}, k_{0}$ and $d_{u_{t_{n}}, k_{0}}$ represent the small-scale fast fading and distance from TU $u_{t_{n}}$ to BS $k_{0}$ on $i$ th subchannel, respectively. The Rayleigh distribution is assumed (i.e., $h_{u_{t},}, k_{0} \sim \mathscr{C} \mathcal{N}(0,1)$ ). From (2), it can be observed that less interference link-gain leads to larger 
allowable transmission power unit, which means that the TU far from the BS is more likely to transmit with larger power.

Thus, the transmission power of TU $u_{t_{n}}$ can be expressed as

$$
P_{u_{t_{n}}}=\min \left\{P L_{u_{t_{n}}}, P_{u_{t_{n}}, \max }\right\}
$$

where $P_{u_{t_{n}} \text {, max }}$ is the maximum transmission power for TU $u_{t_{n}}$ on $i$ th subchannel. Moreover, we can get

$$
P_{u_{t_{n}}}= \begin{cases}P L_{u_{t_{n}}}, & \text { with } P_{b, u_{t_{n}}} \\ P_{u_{t_{n}}, \max }, & \text { with } 1-P_{b, u_{t_{n}}}\end{cases}
$$

where $P_{b, u_{t_{n}}}$ denotes the probability given by

$$
\begin{aligned}
P_{b, u_{t_{n}}} & =\mathbb{P}\left[P_{u_{t_{n}}, \max }>P L_{u_{t_{n}}}\right] \\
& =\mathbb{P}\left[\left|h_{u_{t_{n}}, k_{0}}\right|^{2} d_{u_{t_{n}}, k_{0}}^{-\alpha}>\frac{I_{k_{0}}}{N_{d} P_{u_{t_{n}}, \max }}\right] \\
& =\exp \left(-d_{u_{t_{n}}, k_{0}}^{-\alpha} \frac{I_{k_{0}}}{N_{d} P_{u_{t_{n}}, \max }}\right) .
\end{aligned}
$$

Note that the proposed power control is semidistributed as each TU decides the transmission power according to the allowable transmission power unit broadcasted by the BS and its own maximum power.

\section{Performance Analysis}

In this section, we analyze the proposed scheme in terms of the coverage probability. The performances of the cellular link and D2D links are both analyzed.

4.1. The Cellular Coverage Probability. The uplink SINR from CUE to the BS on $i$ th subchannel can be expressed as [11]

$$
\tilde{\gamma}_{s_{0}, k_{0}}=\frac{P_{s_{0}}\left|h_{s_{0}, k_{0}}\right|^{2} d_{s_{0}, k_{0}}^{-\alpha}}{I_{k_{0}}+\sigma_{k_{0}}^{2}}
$$

where $P_{s_{0}}$ denotes the transmission power of CUE on $i$ th subchannel. $h_{s_{0}, k_{0}}$ and $d_{s_{0}, k_{0}}$ represent the small-scale fast fading and distance from CUE $s_{0}$ to BS $k_{0}$ on $i$ th subchannel, respectively. $h_{s_{0}, k_{0}} \sim \mathscr{C} \mathscr{N}(0,1) . I_{k_{0}}=\sum_{u_{t_{n}} \in \Phi_{\mathrm{DU}}} P_{u_{t_{n}}}\left|h_{u_{t_{n}}, k_{0}}\right|^{2} d_{u_{t_{n}}, k_{0}}^{-\alpha}$ represents the total uplink interference from TU pieces to CUE $s_{0}$ in the BS $k_{0}$ on the $i$ th subchannel. $\sigma_{k_{0}}^{2}$ is the power of the additive Gaussian white noise at $k_{0}$.

Lemma 1. The coverage probability of the cellular link can be expressed as

$$
P_{c c}(\eta)=\mathbb{E}_{d_{s_{0}, k_{0}}}\left\{\exp \left(-A_{1} X_{1}-A_{2} X_{1}^{2 / \alpha}\right)\right\}
$$

where $A_{1}=\sigma_{k_{0}}^{2} \eta, A_{2}=\left(\pi \lambda_{D U} P S_{u_{t_{n}}} \eta^{2 / \alpha} / \sin c(2 / \alpha)\right) \mathbb{E}\left[P_{u_{t_{n}}}^{2 / \alpha}\right]$, $X_{1}=P_{s_{0}}^{-1} d_{s_{0}, k_{0}}^{\alpha}$, and $\eta$ represents the minimum SINR value for reliable uplink connection.
Proof. The coverage probability of the cellular link can be expressed as $[10,12]$

$$
\begin{aligned}
& P_{c c}(\eta)=\mathbb{P}\left(\widetilde{\gamma}_{s_{0}, k_{0}} \geq \eta\right)=\mathbb{P}\left(\frac{P_{s_{0}}\left|h_{s_{0}, k_{0}}\right|^{2} d_{s_{0}, k_{0}}^{-\alpha}}{I_{k_{0}}+\sigma_{k_{0}}^{2}} \geq \eta\right) \\
& =\mathbb{P}\left(\left|h_{s_{0}, k_{0}}\right|^{2} \geq \eta P_{s_{0}}^{-1} d_{s_{0}, k_{0}}^{\alpha}\left(I_{k_{0}}+\sigma_{k_{0}}^{2}\right)\right) \\
& \stackrel{(a)}{=} \mathbb{E}\left[\exp \left(-\eta P_{s_{0}}^{-1} d_{s_{0}, k_{0}}^{\alpha}\left(I_{k_{0}}+\sigma_{k_{0}}^{2}\right)\right)\right] \\
& =\mathbb{E}\left\{e^{-\eta P_{s_{0}}^{-1} d_{s_{0}, k_{0}}^{\alpha} \sigma_{k_{0}}^{2}} e^{\left.-\eta P_{s_{0}}^{-1} d_{s_{0}, k_{0}}^{\alpha} \sum_{u_{t_{n}} \in \Phi_{\mathrm{DU}}} P_{u_{t_{n}}}\left|h_{u_{t_{n}}, k_{0}}\right|^{2} d_{u_{t_{n}}, k_{0}}^{-\alpha}\right\},}\right.
\end{aligned}
$$

where in the equality $(a)$ we use the fact that $\left|h_{s_{0}, k_{0}}\right|^{2} \sim \exp (1)$ [10]. Denote $X_{1}=P_{s_{0}}^{-1} d_{s_{0}, k_{0}}^{\alpha}$. Conditioning on $d_{s_{0}, k_{0}}^{\alpha}$, we can obtain

$$
\begin{aligned}
& \left.\mathbb{P}\left(\widetilde{\gamma}_{s_{0}, k_{0}} \geq \eta\right)\right|_{d_{s_{0}, k_{0}}^{\alpha}} \\
& =e^{-\eta P_{s_{0}}^{-1} d_{s_{0}, k_{0}}^{\alpha} \sigma_{k_{0}}^{2}} e^{-\eta P_{s_{0}}^{-1} d_{s_{0}, k_{0}}^{\alpha} \sum_{u_{t_{n}} \in \Phi_{\mathrm{DU}}} P_{u_{t_{n}}}\left|h_{u_{t_{n}}, k_{0}}\right|^{2} d_{u_{t_{n}}, k_{0}}^{-\alpha}} .
\end{aligned}
$$

The Laplace transform is applied as follows [15]:

$$
\begin{aligned}
L_{\Phi_{D}}(s) & =e^{-s\left(\sum_{u_{t_{n}} \in \Phi_{\mathrm{DU}}} P_{u_{t_{n}}}\left|h_{u_{t_{n}}, k_{0}}\right|^{2} d_{u_{t_{n}}, k_{0}}^{-\alpha}\right)} \\
& =e^{-\left(\pi \lambda_{\mathrm{DU}} P S_{u_{t_{n}}} / \operatorname{sinc}(2 / \alpha)\right) \mathbb{E}\left[P_{u_{t_{n}}}^{2 / \alpha}\right] s^{2 / \alpha}} .
\end{aligned}
$$

Deconditioning with respect to $d_{s_{0}, k_{0}}^{\alpha}$, we combine (9) and (10), and then (7) can be obtained.

According to (7), we can observe that the D2D-related parameter $\mathbb{E}\left[P_{u_{t_{n}}}^{2 / \alpha}\right]$ affects the cellular link coverage probability. In the following, by giving the expression of $\mathbb{E}\left[P_{u_{t_{n}}}^{2 / \alpha}\right]$, we will present how the proposed power control method affects the performance of the cellular link. as

Denote $z=\left|h_{u_{t_{n}},}, k_{0}\right|^{2} d_{u_{t_{n}}}^{-\alpha}, k_{0} ;$ then $\mathbb{E}\left[P_{u_{t_{n}}}^{2 / \alpha}\right]$ can be expressed

$$
\begin{aligned}
\mathbb{E}\left[P_{u_{t_{n}}}^{2 / \alpha}\right] & =\int_{0}^{+\infty} \min \left\{P L_{u_{t_{n}}}^{2 / \alpha}, P_{u_{t_{n}}, \max }^{2 / \alpha}\right\} f(z) d_{z} \\
& =\int_{0}^{+\infty} \min \left\{\left(\frac{I_{k_{0}}}{N_{d} z}\right)^{2 / \alpha}, P_{u_{t_{n}}, \max }^{2 / \alpha}\right\} f(z) d_{z} .
\end{aligned}
$$

Conditioning on the distance $d_{u_{t_{n}}, k_{0}}$ and $I_{k_{0}}, \mathbb{E}\left[P_{u_{t_{n}}}^{2 / \alpha}\right]$ is given by

$$
\begin{aligned}
& \left.\mathbb{E}\left[P_{u_{t_{n}}}^{2 / \alpha}\right]\right|_{d_{u_{t_{n}}, k_{0}}} \\
& =\int_{0}^{+\infty} \min \left\{\left(\frac{I_{k_{0}}}{N_{d} d_{u_{t_{n}}, k_{0}}^{-\alpha} x}\right)^{2 / \alpha}, P_{u_{t_{n}}, \max }^{2 / \alpha}\right\} \\
& \cdot \exp (-x) d_{x} \\
& =\min \left\{\int_{0}^{+\infty}\left(\frac{I_{k_{0}}}{N_{d} d_{u_{t_{n}}, k_{0}}^{-\alpha} x}\right)^{2 / \alpha} \exp (-x) d_{x},\right.
\end{aligned}
$$




$$
\begin{aligned}
& \left.\int_{0}^{+\infty} P_{u_{t_{n}}, \max }^{2 / \alpha} \exp (-x) d_{x}\right\} \\
& =\min \left\{\int_{0}^{+\infty}\left(\frac{I_{k_{0}}}{N_{d} d_{u_{t_{n}}, k_{0}}^{-\alpha} x}\right)^{2 / \alpha} \exp (-x) d_{x},\right. \\
& \left.P_{u_{t_{n}}, \max }^{2 / \alpha}\right\}=\min \left\{\left(\frac{I_{k_{0}}}{N_{d} d_{u_{t_{n}}, k_{0}}^{-\alpha}}\right)^{2 / \alpha} \Gamma\left(\frac{-2+\alpha}{\alpha}\right),\right. \\
& \left.P_{u_{t_{n}}, \max }^{2 / \alpha}\right\},
\end{aligned}
$$

where $\Gamma(x)$ denotes the gamma function. Then, deconditioning with respect to $d_{u_{t_{n}}, k_{0}}$, we can obtain

$$
\begin{aligned}
& \mathbb{E}\left[P_{u_{t_{n}}}^{2 / \alpha}\right]=\min \left\{\mathbb{E}\left[\left(\frac{I_{k_{0}}}{N_{d} d_{u_{t_{n}}, k_{0}}^{-\alpha}}\right)^{2 / \alpha} \Gamma\left(\frac{-2+\alpha}{\alpha}\right)\right],\right. \\
& \left.P_{u_{t_{n}}, \max }^{2 / \alpha}\right\} \\
& =\min \left\{\mathbb{E}\left[\left(\frac{I_{k_{0}}}{N_{d}}\right)^{2 / \alpha} \Gamma\left(\frac{-2+\alpha}{\alpha}\right) d_{u_{t_{n}}, k_{0}}^{2}\right],\right. \\
& \left.P_{u_{t_{n}}, \max }^{2 / \alpha}\right\}=\min \left\{\left(\frac{I_{k_{0}}}{N_{d}}\right)^{2 / \alpha} \Gamma\left(\frac{-2+\alpha}{\alpha}\right) \mathbb{E}\left[d_{u_{t_{n}}, k_{0}}^{2}\right],\right. \\
& \left.P_{u_{t_{n}}, \max }^{2 / \alpha}\right\} \stackrel{(a)}{=} \min \left\{\left(\frac{I_{k_{0}}}{N_{d}}\right)^{2 / \alpha} \Gamma\left(\frac{-2+\alpha}{\alpha}\right) R^{2},\right. \\
& \left.P_{u_{t_{n}}, \max }^{2 / \alpha}\right\} \text {, }
\end{aligned}
$$

where $\mathbb{E}\left[d_{u_{t_{n}}, k_{0}}^{2}\right]=R^{2}$ given in [13]. From (13), we can see that the proposed network-assisted power control method can control the interferences from TU pieces to the CUE accurately by adjusting the parameter $I_{k_{0}}$. The coverage probability of the cellular link decreases with $I_{k_{0}}$. Larger $I_{k_{0}}$ leads to more serious interference.

Generally, we set $I_{k_{0}}$ to keep the uplink SINR of the CUE to be above the target $\eta$. In D2D underlaid cellular networks, the communication link is usually more severely affected by the interference compared with the noise. Hence, we ignore the effect of noise by setting $\sigma_{k_{0}}^{2}=0$ to facilitate the theoretical analysis. In the interference limited regime $\left(\sigma_{k_{0}}^{2}=0\right)$, we have

$$
I_{k_{0}}=\frac{P_{s_{0}}\left|h_{s_{0}, k_{0}}\right|^{2} d_{s_{0}, k_{0}}^{-\alpha}}{\eta} .
$$

Then, plugging (14) into (13) and deconditioning with respect to $\left|h_{s_{0}, k_{0}}\right|^{2}$, it can be obtained that

$$
\begin{aligned}
& \mathbb{E}\left[P_{u_{t_{n}}}^{2 / \alpha}\right]=\min \left\{\mathbb{E}\left[\left(\frac{I_{k_{0}}}{N_{d}}\right)^{2 / \alpha} \Gamma\left(\frac{-2+\alpha}{\alpha}\right) R^{2}\right],\right. \\
& \left.P_{u_{t_{n}}, \max }^{2 / \alpha}\right\}=\min \left\{\mathbb { E } \left[\left(\frac{P_{s_{0}}\left|h_{s_{0}, k_{0}}\right|^{2} d_{s_{0}, k_{0}}^{-\alpha}}{\eta N_{d}}\right)^{2 / \alpha}\right.\right. \\
& \left.\left.\cdot \Gamma\left(\frac{-2+\alpha}{\alpha}\right) R^{2}\right], P_{u_{t_{n}}, \max }^{2 / \alpha}\right\} \\
& \stackrel{(a)}{=} \min \left\{\left(\frac{P_{s_{0}} d_{s_{0}, k_{0}}^{-\alpha}}{N_{d} \eta}\right)^{2 / \alpha} \Gamma\left(\frac{-2+\alpha}{\alpha}\right) \Gamma\left(\frac{2+\alpha}{\alpha}\right) R^{2},\right. \\
& \left.P_{u_{t_{n}}, \max }^{2 / \alpha}\right\},
\end{aligned}
$$

where the equality (a) follows from $\left|h_{s_{0}, k_{0}}\right|^{2} \sim \exp (1)$.

4.2. The Performance of D2D Links. The SINR at RU $u_{r_{n}}$ on $i$ th subchannel can be expressed as [11]

$$
\tilde{\gamma}_{u_{t_{n}}, u_{r_{n}}}=\frac{P_{u_{t_{n}}}\left|h_{u_{t_{n}}, u_{r_{n}}}\right|^{2} d_{u_{t_{n}}, u_{r_{n}}}^{-\alpha}}{I_{D, u_{r_{n}}}+P_{s_{0}}\left|h_{s_{0}, u_{r_{n}}}\right|^{2} d_{s_{0}, u_{r_{n}}}^{-\alpha}+\sigma_{u_{r_{n}}}^{2}},
$$

where $P_{u_{t_{n}}}$ denotes the transmission power of TU $u_{t_{n}}$. $h_{u_{t_{n}}, u_{r_{n}}}$ and $d_{u_{t_{n}}, u_{r_{n}}}$ represent the small-scale fast fading and distance between TU $u_{t_{n}}$ and its corresponding RU $u_{r_{n}}$, respectively. $h_{u_{t_{n}}, u_{r_{n}}} \sim \mathscr{C} \mathcal{N}(0,1) . I_{D, u_{r_{n}}}=$ $\sum_{u_{t_{i}} \in \Phi_{\mathrm{DU}} \backslash\left\{u_{t_{n}}\right\}} P_{u_{t_{i}}}\left|h_{u_{t_{i}}, u_{r_{n}}}\right|^{2} d_{u_{t_{n}}, u_{r_{n}}}^{-\alpha}$ is the total interference from other D2D pairs to RU $u_{r_{n}} . P_{s_{0}}\left|h_{s_{0}, u_{r_{n}}}\right|^{2} d_{s_{0}, u_{r_{n}}}^{-\alpha}$ is the interference from the CUE $s_{0}$ to RU $u_{r_{n}} . \sigma_{u_{r_{n}}}^{2}$ is the power of the additive Gaussian white noise at $u_{r_{n}}$.

Lemma 2. Conditioning on the distance $d_{u_{t_{n}}, u_{t_{n}}}$, the coverage probability of $R U u_{r_{n}}$ in the interference limited regime $\left(\sigma_{u_{r_{n}}}^{2}=\right.$ 0) is given by

$$
\begin{aligned}
& \left.P_{d c}(\beta)\right|_{d_{u_{t_{n}}, u_{r_{n}}}} \\
& \quad \approx \mathbb{E}_{P_{u_{t_{n}}}}\left[\exp \left(-B_{1} P_{u_{t_{n}}}^{-2 / \alpha}\right) \frac{1}{1+B_{2} P_{u_{t_{n}}}^{-2 / \alpha}}\right]
\end{aligned}
$$


where $B_{1}=\left(\pi \lambda_{D U} P S_{u_{n}} \beta^{2 / \alpha} / \sin c(2 / \alpha)\right) \mathbb{E}\left[P_{u_{t_{n}}}^{2 / \alpha}\right] d_{u_{t_{n}}, u_{r_{n}}}^{2}, B_{2}=$ $\left(\beta P_{s_{0}}\right)^{2 / \alpha}\left((45 \pi)^{2} d_{u_{t_{n}}, u_{v_{n}}}^{2} /(128 R)^{2}\right)$, and $\beta$ represents the minimum SINR value for reliable D2D connection.

Proof. The coverage probability of RU $u_{r_{n}}$ can be expressed as $[10,12]$

$$
\begin{aligned}
& \left.P_{d c}(\beta)\right|_{d_{u_{t_{n}}, u_{r_{n}}}}=\left.\mathbb{P}\left(\tilde{\gamma}_{u_{t_{1}}, u_{t_{1}}} \geq \beta\right)\right|_{d_{u_{t_{n}}, u_{r_{n}}}} \\
& =\mathbb{P}\left(\frac{P_{u_{t_{n}}}\left|h_{u_{t_{n}}, u_{r_{n}}}\right|^{2} d_{u_{t_{n}}, u_{r_{n}}}^{-\alpha}}{I_{D, u_{r_{n}}}+P_{s_{0}}\left|h_{s_{0}, u_{r_{n}}}\right|^{2} d_{s_{0}, u_{r_{n}}}^{-\alpha}} \geq \beta\right) \\
& =\mathbb{P}\left(\left|h_{u_{t_{1}}, u_{t_{1}}}\right|^{2}\right.
\end{aligned}
$$

$$
\begin{aligned}
& \left.\geq P_{u_{t_{n}}}^{-1} d_{u_{t_{n}}, u_{r_{n}}}^{\alpha} \beta\left(I_{D, u_{r_{n}}}+P_{s_{0}}\left|h_{s_{0}, u_{r_{n}}}\right|^{2} d_{s_{0}, u_{r_{n}}}^{-\alpha}\right)\right) \\
& =\mathbb{E}\left[e^{-P_{u_{t_{n}}}^{-1} d_{u_{t_{n}}, u_{r_{n}}}^{\alpha} \beta\left(I_{D, u_{r_{n}}}+P_{s_{0}}\left|h_{s_{0}, u_{r_{n}}}\right|^{2} d_{s_{0}, u_{r_{n}}}^{-\alpha}\right)}\right] .
\end{aligned}
$$

We use Slivnyak's theorem [16] as follows:

$$
\begin{aligned}
L_{\Phi_{\mathrm{DU}} \backslash\left\{u_{t_{n}}\right\}}(s) & =\mathbb{E}\left[e^{\left.-s \sum_{u_{t_{i}} \in \Phi_{\mathrm{DU}} \backslash\left\{u_{t_{n}}\right\}} P_{u_{t_{i}}}\left|h_{u_{t_{i}}, u_{r_{n}}}\right|^{2} d_{u_{t_{n}}, u_{r_{n}}}^{-\alpha}\right]}\right. \\
& =L_{\Phi_{\mathrm{DU}}}(s)=e^{-\left(\pi \lambda_{\mathrm{DU}} / \sin c(2 / \alpha)\right) \mathbb{E}\left[P_{u_{t_{n}}}^{2 / \alpha}\right] s^{2 / \alpha}} .
\end{aligned}
$$

Conditioned on $P_{u_{t_{n}}},(18)$ can be written as

$$
\left.P_{d c}(\beta)\right|_{d_{u_{t_{n}}, u_{r_{n}}}}=e^{-\left(\pi \lambda_{\mathrm{DU}} \beta^{2 / \alpha} / \operatorname{sinc}(2 / \alpha)\right) \mathbb{E}\left[P_{u_{t_{n}}}^{2 / \alpha}\right] d_{u_{t_{n}}, u_{r_{n}}}^{2} P_{u_{t_{n}}}^{-2 / \alpha}} \mathbb{E}\left[e^{-P_{u_{t_{n}}}^{-1} d_{u_{t_{n}}, u_{r_{n}}}^{\alpha} \beta P_{s_{0}}\left|h_{s_{0}, u_{r_{n}}}\right|^{2} d_{s_{0}, u_{r_{n}}}^{-\alpha}}\right]
$$

where $\mathbb{E}\left[e^{\left.-P_{u_{t_{n}}}^{-1} d_{u_{t_{n}}, u_{r_{n}}}^{\alpha} \beta P_{s_{0}}\left|h_{s_{0}, u_{r_{n}}}\right|^{2} d_{s_{0}, u_{r_{n}}}^{-\alpha}\right]}=\mathbb{E}_{d_{s_{0}, u_{r_{n}}}}[1 /(1+\right.$ $\left.\left.\beta P_{s_{0}} P_{u_{t_{n}}}^{-1} d_{u_{t_{n}}, u_{r_{n}}}^{\alpha} d_{s_{0}, u_{r_{n}}}^{-\alpha}\right)\right]$

Furthermore, we use the approximation $\mathbb{E}_{d_{s_{0}, u_{r_{n}}}}[1 /(1+$ $\left.\left.k / d_{s_{0}, u_{r_{n}}}^{\alpha}\right)\right] \simeq\left[1 /\left(1+k^{2 / \alpha} / \mathbb{E}\left[d_{s_{0}, u_{r_{n}}}\right]^{2}\right)\right]$ in $[10]$ and $\mathbb{E}\left[d_{s_{0}, u_{r_{n}}}\right]=$ $128 R / 45 \pi$ in [13]. Equation (20) can be expressed as in (17).

In (17), we can see that the coverage probability of the D2D link is determined by two factors: (a) the total interference power created by other active D2D links (i.e., $B_{1} P_{u_{t_{n}}}^{-2 / \alpha}$ ) and (b) the approximated effect of the uplink interference from the CUE (i.e., $\left.1 /\left(1+B_{2} P_{u_{t_{n}}}^{-2 / \alpha}\right)\right)$.

Unlike intuition, it is interesting to find that the coverage probability of the D2D link does not necessarily increase with the parameter $I_{k_{0}}$ for any given $d_{u_{t_{n}}, u_{r_{n}}}, d_{u_{t_{n}}, k_{0}}$, and $\left|h_{u_{t_{n}}, k_{0}}\right|^{2}$.

$$
\begin{aligned}
& \left.P_{d c}(\beta)\right|_{d_{u_{t_{n}}, u_{u_{n}}}, d_{u_{t_{n}}, k_{0}}, h_{u_{t_{n}}, k_{0}}} \\
& \quad \approx \exp \left(-\frac{\pi \lambda_{\mathrm{DU}} P S_{u_{n}} \beta^{2 / \alpha} \mathbb{E}\left[P_{u_{t_{n}}}^{2 / \alpha}\right]}{\sin c(2 / \alpha)} d_{u_{t_{n}}, u_{r_{n}}}^{2 / \alpha}\right) \\
& \cdot \frac{1}{1+\left(\beta\left(P_{s_{0}} / P_{u_{t_{n}}}\right)\right)^{2 / \alpha}\left((45 \pi)^{2} d_{u_{t_{n}}, u_{r_{n}}}^{2} /(128 R)^{2}\right)} .
\end{aligned}
$$

According to (3), (13), and (21), there are four cases as follows:

(1) $\left(I_{k_{0}} / N_{d}\right)^{2 / \alpha} \Gamma((-2+\alpha) / \alpha) R^{2} \leq P_{u_{t_{n}}, \max }^{2 / \alpha}$ and $P_{u_{t_{n}} \text {, max }}^{2 / \alpha} \leq$ $\left(I_{k_{0}} / N_{d}\right)^{2 / \alpha}\left(\left|h_{u_{t_{n}}, k_{0}}\right|^{-2}\right)^{2 / \alpha} d_{u_{t_{n}}, k_{0}}^{2}, P_{d c}(\beta)$ decrease with $I_{k_{0}}$.

(2) $\left(I_{k_{0}} / N_{d}\right)^{2 / \alpha} \Gamma((-2+\alpha) / \alpha) R^{2} \leq P_{u_{t_{n}}, \max }^{2 / \alpha}$ and $\left(I_{k_{0}} /\right.$ $\left.N_{d}\right)^{2 / \alpha}\left(\left|h_{u_{t_{n}}, k_{0}}\right|^{-2}\right)^{2 / \alpha} d_{u_{t_{n}}, k_{0}}^{2} \leq P_{u_{t_{n}}, \max }^{2 / \alpha}, P_{d c}(\beta)$ increase with $I_{k_{0}}$.
(3) $P_{u_{t_{n}}, \max }^{2 / \alpha} \leq\left(I_{k_{0}} / N_{d}\right)^{2 / \alpha} \Gamma((-2+\alpha) / \alpha) R^{2}$ and $P_{u_{t_{n}}, \max }^{2 / \alpha} \leq$ $\left(I_{k_{0}} / N_{d}\right)^{2 / \alpha}\left(\left|h_{u_{t_{n}}, k_{0}}\right|^{-2}\right)^{2 / \alpha} d_{u_{t_{n}}, k_{0}}^{2}, P_{d c}(\beta)$ donot change with $I_{k_{0}}$.

(4) $P_{u_{t_{n}}, \max }^{2 / \alpha} \leq\left(I_{k_{0}} / N_{d}\right)^{2 / \alpha} \Gamma((-2+\alpha) / \alpha) R^{2}$ and $\left(I_{k_{0}} /\right.$ $\left.N_{d}\right)^{2 / \alpha}\left(\left|h_{u_{t_{n}}, k_{0}}\right|^{-2}\right)^{2 / \alpha} d_{u_{t_{n}}, k_{0}}^{2} \leq P_{u_{t_{n}}, \max }^{2 / \alpha}, P_{d c}(\beta)$ increase with $I_{k_{0}}$.

For case (1), the channel gain from TU $u_{t_{n}}$ to BS $k_{0}$ is small; TU $u_{t_{n}}$ can transmit with the maximum transmission power $P_{u_{t_{n}} \text {, max }}$. However, for other TU pieces, the transmission powers are limited by $I_{k_{0}}$. When $I_{k_{0}}$ increases, the transmission power of TU $u_{t_{n}}$ does not change and the average transmission power of other TU pieces (i.e., $\mathbb{E}\left[P_{u_{t_{n}}}^{2 / \alpha}\right]$ ) increases, which reduces the coverage probability of the D2D $\operatorname{link}\left(u_{t_{n}}, u_{r_{n}}\right)$.

\section{Optimizing D2D On-Off Threshold}

In this section, we assume that each TU has the same maximum transmission power (i.e., $P_{u_{t_{n}}, \max }=P_{u, \max }$ ). In the following, we first introduce the area spectral efficiency of D2D links and the cellular link. Second, the upper bound of the area spectral efficiency of D2D links and the lower bound of the area spectral efficiency of the cellular link are obtained, respectively. Third, we optimize the D2D on-off threshold by maximizing the approximated total area spectral efficiency.

Since the analysis performed for a typical link indicates the spatially averaged performance of the network by Slivnyak's theorem $[16,17]$, for any given $d_{u_{t_{n}}, u_{r_{n}}}, d_{u_{t_{n}}, k_{0}}$, and $\left|h_{u_{t_{n}}, k_{0}}\right|^{2}$, the area spectral efficiency of D2D communications is given as $[12,18]$ 


$$
\begin{aligned}
& \Upsilon^{D}(\beta)=\lambda_{\mathrm{DU}} P S_{u_{n}} \log _{2}(1+\beta) \mathbb{P}\left(\widetilde{\gamma}_{u_{t_{n}}, u_{r_{n}}} \geq \beta\right) \\
& \approx \lambda_{\mathrm{DU}} P S_{u_{n}} \\
& \cdot \exp \left(-\frac{\pi \lambda_{\mathrm{DU}} P S_{u_{n}} \beta^{2 / \alpha}}{\sin c(2 / \alpha)} \frac{\mathbb{E}\left[P_{u_{t_{n}}}^{2 / \alpha}\right]}{P_{u_{t_{n}}}^{2 / \alpha}} d_{u_{t_{n}}, u_{r_{n}}}^{2}\right) \\
& \cdot \frac{\log _{2}(1+\beta)}{1+\left(\beta\left(P_{s_{0}} / P_{u_{t_{n}}}\right)\right)^{2 / \alpha}\left((45 \pi)^{2} d_{u_{t_{n}}, u_{r_{n}}}^{2} /(128 R)^{2}\right)} .
\end{aligned}
$$
[10]:

The area spectral efficiency of the cellular link is as follows

$$
\Upsilon^{C}(\eta)=\frac{\log 2(1+\eta) P_{c c}(\eta)}{\left(\pi R^{2}\right)}
$$

According to (22) and (23), the optimal access probability for D2D links can be obtained by solving the following optimization problem:

$$
\begin{aligned}
\max _{P S_{u_{t_{n}}}} & \omega_{1} \Upsilon^{C}(\eta) \\
\max _{P S_{u_{t_{n}}}} & \omega_{2} \Upsilon^{D}(\beta) \\
\text { s.t. } & 0<P S_{u_{t_{n}}}<1,
\end{aligned}
$$

where $\omega_{1}$ and $\omega_{2}$ are the introduced scalar weights, which represent the priorities of CUE and DUE pieces, respectively. Note that $\Upsilon^{\text {total }}(\eta, \beta)=\omega_{1} \Upsilon^{C}(\eta)+\omega_{2} \Upsilon^{D}(\beta)$ can represent the total area spectral efficiency including both the cellular link and D2D links. Note that it is difficult to obtain the closed-form solution of the original optimization problem. Therefore, in order to facilitate the analysis and reduce the computational complexity, we introduce the upper bound of the area spectral efficiency of D2D links and the lower bound of the area spectral efficiency of the cellular link.

Lemma 3. The upper bound of the area spectral efficiency of D2D communications is obtained as

$$
\Upsilon^{D}(\beta) \leq \Upsilon_{u p p e r}^{D}(\beta)
$$

with

$$
\begin{aligned}
& Y_{\text {upper }}^{D}(\beta) \\
& = \begin{cases}\lambda_{D U} P S_{u_{n}} D_{2} \exp \left(-D_{1}\right), & \text { for }\left(\frac{P_{s_{0}} d_{s_{0}, k_{0}}^{-\alpha}}{N_{d} \eta}\right)^{2 / \alpha} \Gamma\left(\frac{-2+\alpha}{\alpha}\right) \Gamma\left(\frac{2+\alpha}{\alpha}\right) R^{2} \geq P_{u, \text { max }}^{2 / \alpha} \\
\lambda_{D U} P S_{u_{n}} D_{2} \exp \left(-\frac{D_{1}\left(P_{s_{0}} d_{s_{0}, k_{0}}^{-\alpha} / N_{d} \eta\right)^{2 / \alpha} \Gamma((-2+\alpha) / \alpha) \Gamma((2+\alpha) / \alpha) R^{2}}{P_{u, \text { max }}^{2 / \alpha}}\right), & \text { for }\left(\frac{P_{s_{0}} d_{s_{0}, k_{0}}^{-\alpha}}{N_{d} \eta}\right)^{2 / \alpha} \Gamma\left(\frac{-2+\alpha}{\alpha}\right) \Gamma\left(\frac{2+\alpha}{\alpha}\right) R^{2}<P_{u, \text { max }}^{2 / \alpha},\end{cases}
\end{aligned}
$$

where $D_{1}=\left(\pi \lambda_{D U} P S_{u_{n}} \beta^{2 / \alpha} / \sin c(2 / \alpha)\right) d_{u_{t_{n}}, u_{r_{n}}}^{2}, D_{2}=\log _{2}(1+$ $\beta) /\left(1+\left(\beta\left(P_{s_{0}} / P_{u, \max }\right)\right)^{2 / \alpha}\left((45 \pi)^{2} d_{u_{t_{n}}, u_{r_{n}}}^{2} /(128 R)^{2}\right)\right)$.

Proof. See Appendix A.

Conditioning on the distance $d_{s_{0}, k_{0}}$, we also compute a lower bound of the coverage probability of the cellular link by setting $\mathbb{E}\left[P_{u_{t_{n}}}^{2 / \alpha}\right]=P_{u \text {, max }}^{2 / \alpha}$ in the interference limited regime $\left(\sigma_{k_{0}}^{2}=0\right)$, which can be expressed as

$$
P_{c c, \text { low }}(\eta)=\exp \left(-\frac{\pi \lambda_{\mathrm{DU}} P S_{u_{t_{n}}} \eta^{2 / \alpha}}{\sin c(2 / \alpha)} \frac{P_{u, \max }^{2 / \alpha}}{P_{s_{0}}^{2 / \alpha}} d_{s_{0}, k_{0}}^{2}\right) .
$$

The lower bound of the area spectral efficiency of the cellular link is given by

$$
\Upsilon_{\text {low }}^{C}(\eta)=\frac{\log 2(1+\eta) P_{c c, \text { low }}(\eta)}{\left(\pi R^{2}\right)}
$$

According to (25) and (28), original problem (24) can be approximated as follows:

$$
\begin{aligned}
\max _{P S_{u_{t_{n}}}} & \omega_{1} \Upsilon_{\text {low }}^{C}(\eta) \\
\max _{P S_{u_{t_{n}}}} & \omega_{2} \Upsilon_{\text {upper }}^{D}(\beta) \\
\text { s.t. } & 0<P S_{u_{t_{n}}}<1
\end{aligned}
$$

which is a multicriteria optimization problem. According to (25) and (28), $\Upsilon_{\text {low }}^{C}(\eta)$ decreases with $P S_{u_{t_{n}}}$, while $\Upsilon_{\text {upper }}^{D}(\beta)$ is nonmonotonic with $P S_{u_{t_{n}}}$. Generally, it is difficult to maximize the two objectives simultaneously. In order to balance these two objectives, the weighted sum method in multicriteria optimization is used to obtain the Pareto optimal solution [19]. Then, the multicriteria optimization with high complexity can be converted into a simpler single objective optimization problem given by

$$
\begin{aligned}
\max _{P S_{u_{t_{n}}}} & \omega_{1} \Upsilon_{\text {low }}^{C}(\eta)+\omega_{2} \Upsilon_{\text {upper }}^{D}(\beta) \\
\text { s.t. } & 0<P S_{u_{t_{n}}}<1 .
\end{aligned}
$$

The solution of (30) is a Pareto optimal solution to the optimization problem of (29) [19]. 
Theorem 4. For the optimization problem

$$
\begin{array}{ll}
\max : & f(x)=\varepsilon_{1} e^{-a x}+\varepsilon_{2} x e^{-b x} \\
\text { s.t. } & 0<x<1
\end{array}
$$

with $\varepsilon_{1} \geq 0, \varepsilon_{2} \geq 0, a>0$, and $b>0$, the optimal solution $x^{*}$ is obtained as

$$
x_{o p t}=\max \{\min \{\widehat{x}, 1\}, 0\}
$$

with

$$
\widehat{x}=\frac{1}{b}+\frac{1}{q} \omega\left(-\frac{p q e^{-q / b}}{b}\right),
$$

$$
\begin{aligned}
& \phi_{1}=\frac{\rho_{1} \varepsilon_{1}}{\varepsilon_{2}} \\
& \phi_{2}=\rho_{1}-\rho_{2} \\
& \varepsilon_{1}=\omega_{1} \frac{\log _{2}(1+\eta)}{\pi R^{2}}, \\
& \rho_{1}=\frac{\pi \lambda_{\mathrm{DU}} \eta^{2 / \alpha}}{\sin c(2 / \alpha)}\left(\frac{P_{u, \max }}{P_{s_{0}}}\right)^{2 / \alpha} d_{s_{0}, k_{0}}^{2}, \\
& \varepsilon_{2}=\frac{\omega_{2} \lambda_{\mathrm{DU}} \log _{2}(1+\beta)}{1+\left(\beta\left(P_{s_{0}} / P_{u, \max }\right)\right)^{2 / \alpha}\left((45 \pi)^{2} d_{u_{t_{n}}, u_{v_{n}}}^{2} /(128 R)^{2}\right)},
\end{aligned}
$$

where $p=a \varepsilon_{1} / \varepsilon_{2}, q=a-b$, and $\omega(x)$ denotes the Lambert $\omega$ function defined as the inverse function of $g(x)=x e^{x}$.

Proof. See Appendix B.

According to Theorem 4, the optimal access threshold can be obtained as

$$
P S_{u_{t_{n}}}^{*}=\max \left\{\min \left\{\hat{P S_{u_{t_{n}}}}, 1\right\}, 0\right\}
$$

with

$$
\stackrel{\wedge}{P S}_{u_{t_{n}}}=\frac{1}{\rho_{2}}+\frac{1}{\phi_{2}} \omega\left(-\frac{\phi_{1} \phi_{2} e^{-\phi_{2} / \rho_{2}}}{\rho_{2}}\right),
$$

where

$\rho_{2}$

$$
=\left\{\begin{array}{lr}
\frac{\pi \lambda_{\mathrm{DU}} \beta^{2 / \alpha}}{\sin c(2 / \alpha)} d_{u_{t_{n}}, u_{r_{n}}{ }^{\prime}}^{2} & \text { for }\left(\frac{P_{s_{0}} d_{s_{0}, k_{0}}^{-\alpha}}{N_{d} \eta}\right)^{2 / \alpha} \Gamma\left(\frac{-2+\alpha}{\alpha}\right) \Gamma\left(\frac{2+\alpha}{\alpha}\right) R^{2} \geq P_{u, \text { max }}^{2 / \alpha} \\
\frac{\pi \lambda_{\mathrm{DU}} \beta^{2 / \alpha}\left(P_{s_{0}} d_{s_{0}, k_{0}}^{-\alpha} / N_{d} \eta\right)^{2 / \alpha} \Gamma((-2+\alpha) / \alpha) \Gamma((2+\alpha) / \alpha) R^{2}}{\sin c(2 / \alpha)} d_{u_{t_{n}}, u_{r_{n}},}^{2}, & \text { for }\left(\frac{P_{s_{0}} d_{s_{0}, k_{0}}^{-\alpha}}{N_{d} \eta}\right)^{2 / \alpha} \Gamma\left(\frac{-2+\alpha}{\alpha}\right) \Gamma\left(\frac{2+\alpha}{\alpha}\right) R^{2}<P_{u, \text { max }}^{2 / \alpha} .
\end{array}\right.
$$

Then, the optimal access threshold is designed according to the optimal access probability. According to (1) and (34), the optimal access threshold for the $\mathrm{D} 2 \mathrm{D}$ pair $u_{n}$ is given as

$$
G_{\min , u_{t_{n}}}^{*}=\frac{-\ln \left(P S_{u_{t_{n}}}^{*}\right)}{d_{u_{t_{n}}, u_{v_{n}}}^{\alpha}} .
$$

One D2D pair decides whether to access the current subchannel based solely on the knowledge of its own communication distance, channel gain, and the optimal access threshold. In order to calculate the access threshold, each TU needs some system parameters (e.g., $\lambda_{\mathrm{DU}}, P_{s_{0}}$, and $d_{s_{0}, k_{0}}$ ). Compared with DUE pieces, the BS is more convenient for obtaining these parameters and broadcasting them to each TU. Then, each D2D pair can calculate its own optimal access threshold according to (34) and (37) based on the system parameters and $d_{u_{t_{n}}, u_{r_{n}}}$ independently. $d_{u_{t_{n}}, u_{r_{n}}}$ can be easily known by the associated TU $u_{t_{n}}$. Since (34) and (37) have the closed-forms, the computational burden is quite low for each TU.

Note that the parameter $\rho_{2}$ in (35) has two possible expressions due to the relationship between $P_{u, \max }^{2 / \alpha}$ and $\left(P_{s_{0}} d_{s_{0}, k_{0}}^{-\alpha} / N_{d} \eta\right)^{2 / \alpha} \Gamma((-2+\alpha) / \alpha) \Gamma((2+\alpha) / \alpha) R^{2}$. However, the number of active TU pieces (i.e., $N_{d}$ ) cannot be known before all the D2D pairs decide whether to access the current subchannel. In this paper, we average over the past subchannels as in Algorithm 1 and apply $\widehat{N}_{d, i}$ as the estimation of $N_{d, i}$ that is used to calculate $\rho_{2}$ in (35).

Though $\widehat{N}_{d, i}$ is not the real number of active TU pieces on the subchannel $i$, the optimal access threshold based on $\widehat{N}_{d, i}$ can achieve a good performance, which will be verified by the extensive simulations in the following. This is because $\widehat{N}_{d, i}$ represents the statistical average of $N_{d, i}$ and $\widehat{N}_{d, i}$ is close to $N_{d, i}$ with high D2D link density $\lambda_{\mathrm{DU}}$. 
(1) Initial set: $\widehat{N}_{d, 1}=N_{d}^{\text {tol }}$

(2) For $i=2: M$

(3) $\widehat{N}_{d, i}=\left(\widehat{N}_{d, 1}+\cdots+\widehat{N}_{d, i-1}\right) /(i-1)$ is used to calculate the optimal access as in Eq. (25) and Eq. (34).

(4) Count the real number of active $N_{d, i}$ on $i$ th sub-channel.

(5) $\widehat{N}_{d, i}=N_{d, i}$

(6) end

Algorithm 1: Estimating the number of active TUs for optimal access threshold.

\section{Simulation Results}

To evaluate the proposed scheme, we carry out extensive simulations by the Matlab tool and discuss the results in this section. The path-loss exponent $\alpha=4$. We assume the average radius of a cell is $500 \mathrm{~m}$; that is, $R=500 \mathrm{~m}$. The BS is located at the center and the CUE is uniformly located in the cell of radius $R$. The transmit power of CUE $P_{s_{0}}=$ $100 \mathrm{~mW}$. Given the D2D communication range $R_{d}=50 \mathrm{~m}$, each intended RU is uniformly and independently located within $\mathrm{Rd}$ of its associated TU in the isotropic direction. The maximum transmit power of TU pieces $P_{u_{t_{n}}, \max }=P_{u, \max }=$ $0.1 \mathrm{~mW}$. The scalar weight factor for the DUE is $\omega_{2}=1$. The performance of the proposed method is compared with three other strategies:

(1) NADD: network-assisted, device-decided scheme [11]

(2) SFPC: statistical features-based power control scheme [12]

(3) NPC: all D2D pairs access the current subchannel. There is no power control and all TU pieces transmit with maximum transmit power $P_{u, \max }$

(4) Proposed: the proposed method.

In Figure 4, the area spectral efficiency performances versus target SIR threshold according to different power control methods are shown. We can see that the proposed method improves the area spectral efficiency compared to other algorithms, especially in the high target SIR regime. This implies that the proposed method is efficient at mitigating both intra-D2D and cross-tier interference when D2D links communicate with a high data rate. Moreover, it is interesting to see that the NADD method is even worse than NPC in terms of the area spectral efficiency. This is because though the NADD method guarantees the performance of the CUE, the NADD method leads to a sharp performance fall for D2D links, which results in the area spectral efficiency decrease in the end.

Figure 5 shows the coverage probabilities of CUEs and DUE pieces versus target SIR threshold according to different power control methods. In Figure 5, it can be observed that the proposed method achieves the best performance for both the CUE and DUE pieces in terms of the coverage probability in the high target SIR regime. Although the proposed method does not have the highest coverage probabilities for the CUE and DUE pieces in the low target SIR regime (e.g., the target

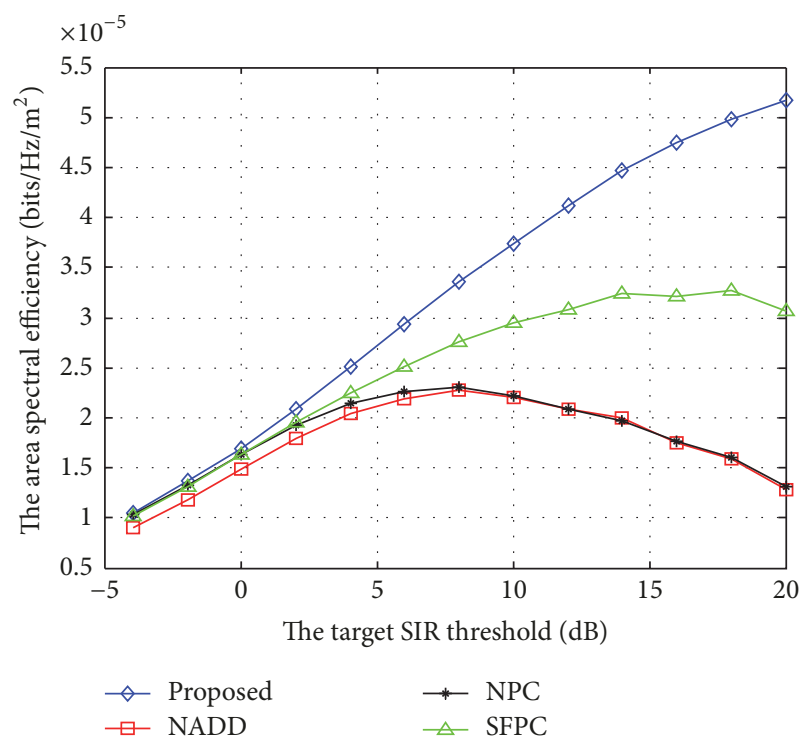

FIGURE 4: The area spectral efficiency $\Upsilon^{\text {total }}(\beta)$ versus target SIR threshold according to different strategies with $\eta=\beta, \lambda_{\mathrm{DU}}=3 \times$ $10^{-5}$, and $\omega_{1}=10$.

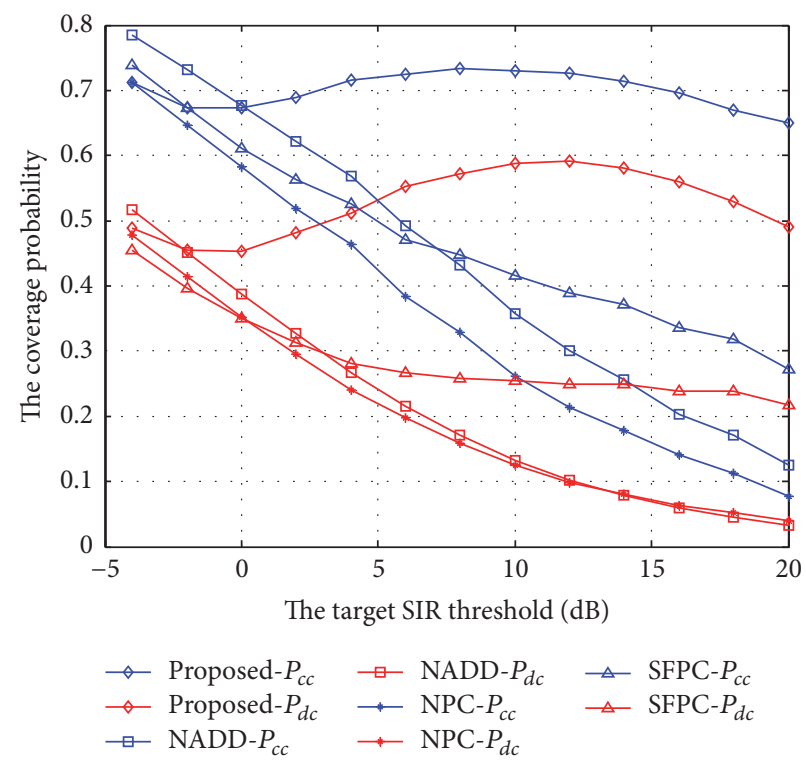

FIGURE 5: Coverage probability performance of both the CUE and the DUE according to different strategies with $\eta=\beta, \lambda_{\mathrm{DU}}=3 \times 10^{-5}$, and $\omega_{1}=10$. 


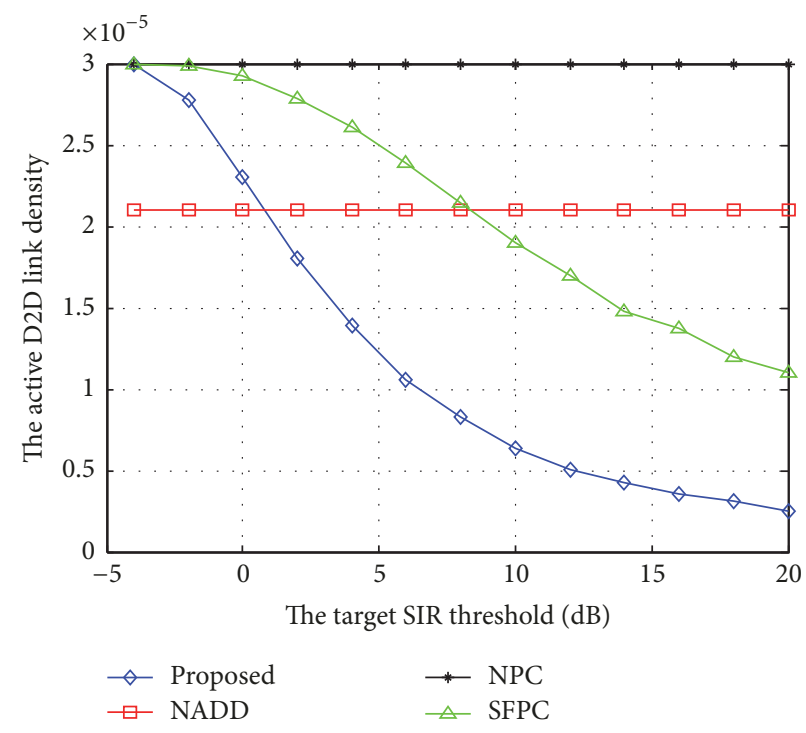

FIGURE 6: The active D2D link density versus target SIR threshold according to different strategies with $\eta=\beta, \lambda_{\mathrm{DU}}=3 \times 10^{-5}$, and $\omega_{1}=10$.

SIR $=-4 \mathrm{~dB}$ ), it can achieve the best area spectral efficiency as shown in Figure 4. This is because there are more active DUE pieces for the proposed scheme as shown in Figure 6.

In Figure 6, the active D2D link density versus target SIR threshold is shown. We can observe that the active D2D link densities of the proposed method and the SFPC decrease with the target SIR threshold, and the active D2D link densities of the NADD and NPC do not change with the target SIR threshold. On the one hand, compared with the NADD and NPC, the proposed method and the SFPC can limit the active D2D link density to guarantee the area spectral efficiency performance. On the other hand, though the active D2D link density of the proposed method is lower than the other methods in the high target SIR regime, the proposed method can achieve the best area spectral efficiency performance. This is because the proposed method can achieve the best performance for both the CUE and DUE pieces in terms of the coverage probability in the high target SIR regime.

Figure 7 plots the area spectral efficiency versus D2D link density $\lambda_{\mathrm{DU}}$. The proposed method can improve the area spectral efficiency significantly compared to other schemes. With the increase of $\lambda_{\mathrm{DU}}$, for NADD and NPC, the area spectral efficiency decreases. This is because the number of active DUE pieces increases with $\lambda_{\mathrm{DU}}$ and the intraD2D and cross-tier interferences become more serious. For the proposed scheme, the area spectral efficiency does not decrease with $\lambda_{\mathrm{DU}}$ according to the optimal threshold that balances the performances of the CUE and DUE pieces.

In Figures 8 and 9, the numbers in the $x$-axis represent different schemes as follows: (1) the proposed method; (2) NADD; (3) NPC; (4) SFPC. Figures 8 and 9 show $\omega_{1} \Upsilon^{C}(\beta)$ and $\omega_{2} \Upsilon^{D}(\beta)$ according to $\omega_{1}=5$ and $\omega_{1}=15$, respectively. Comparing Figure 8 with Figure 9 , we can see that the percentage of $\omega_{1} \Upsilon^{C}(\beta)$ for the proposed scheme increases

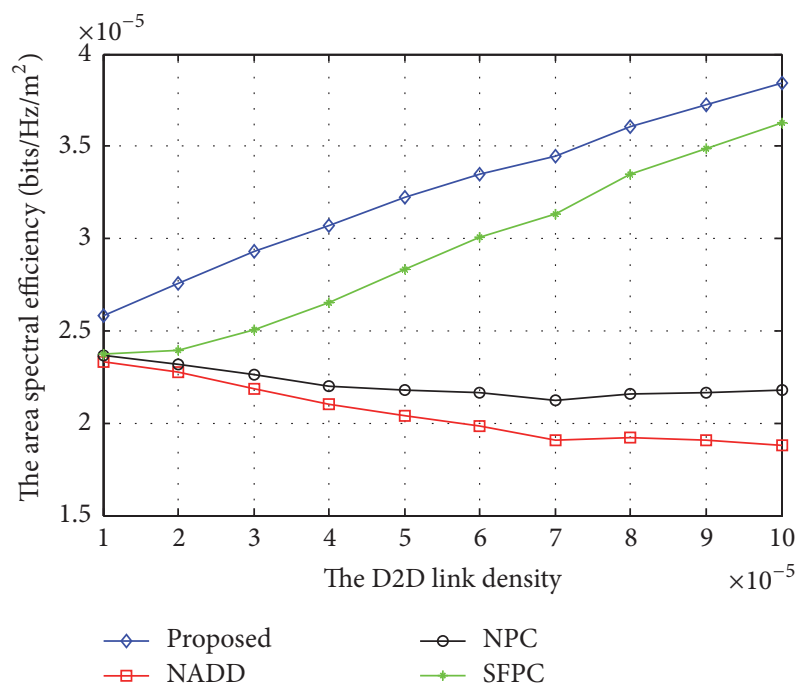

Figure 7: The area spectral efficiency $\Upsilon^{\text {total }}(\beta)$ versus D2D link density $\lambda_{\mathrm{DU}}$ according to different strategies with $\eta=\beta=6 \mathrm{~dB}$ and $\omega_{1}=10$.

with $\omega_{1}$. This is because the priority of the CUE increases with $\omega_{1}$ and the larger percentage of $\omega_{1} \Upsilon^{C}(\beta)$ leads to a larger total area spectral efficiency. While the percentage of $\omega_{1} \Upsilon^{C}(\beta)$ for the other schemes also increases, the improved range of the proposed scheme is the largest, leading to greatest performance improvement. Moreover, the performance of NADD (case (2)) is worse than NPC (case (3)) for $\omega_{1}=5$, while it is opposite for $\omega_{1}=15$. This is because NADD can guarantee the performance of the CUE. When the priority of the CUE increases with $\omega_{1}$, the performance of NADD becomes better than NPC in terms of total area spectral efficiency. 

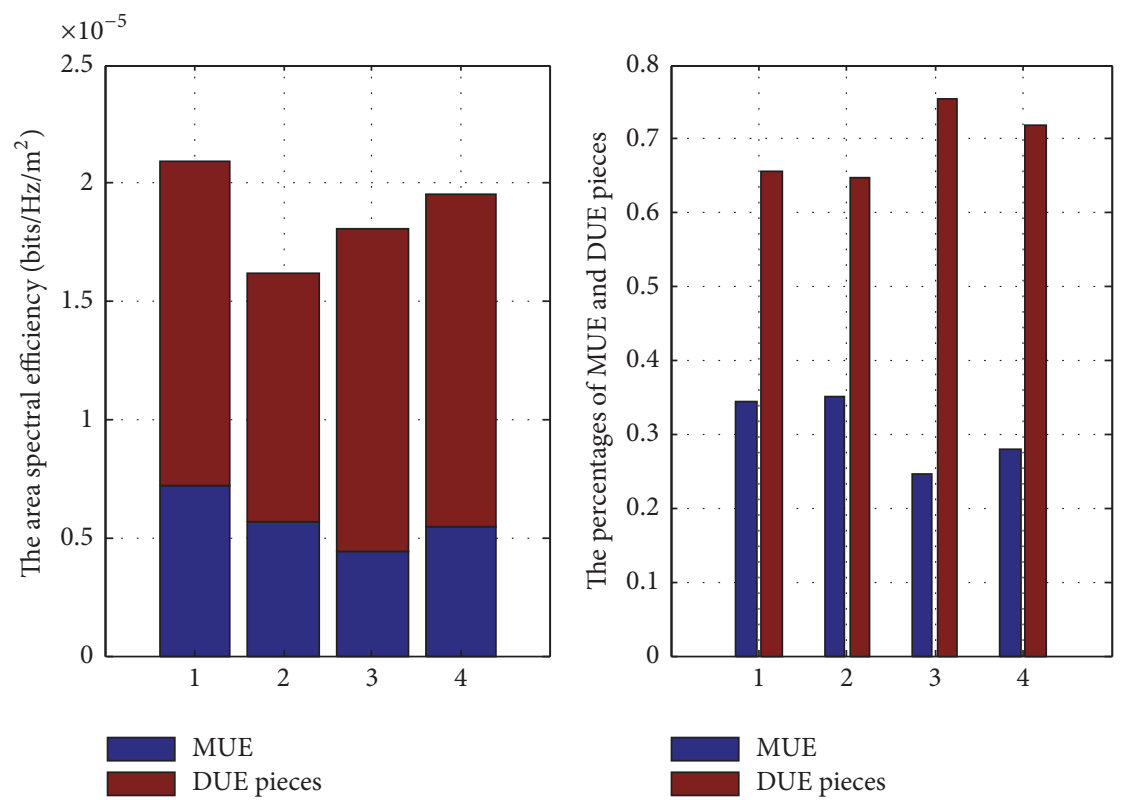

FIgURE 8: The figure shows $\omega_{1} \Upsilon^{C}(\beta)$ and $\omega_{2} \Upsilon^{D}(\beta)$ with $\omega_{1}=5, \eta=\beta=6 \mathrm{~dB}$, and $\lambda_{\mathrm{DU}}=3 \times 10^{-5}$, respectively.
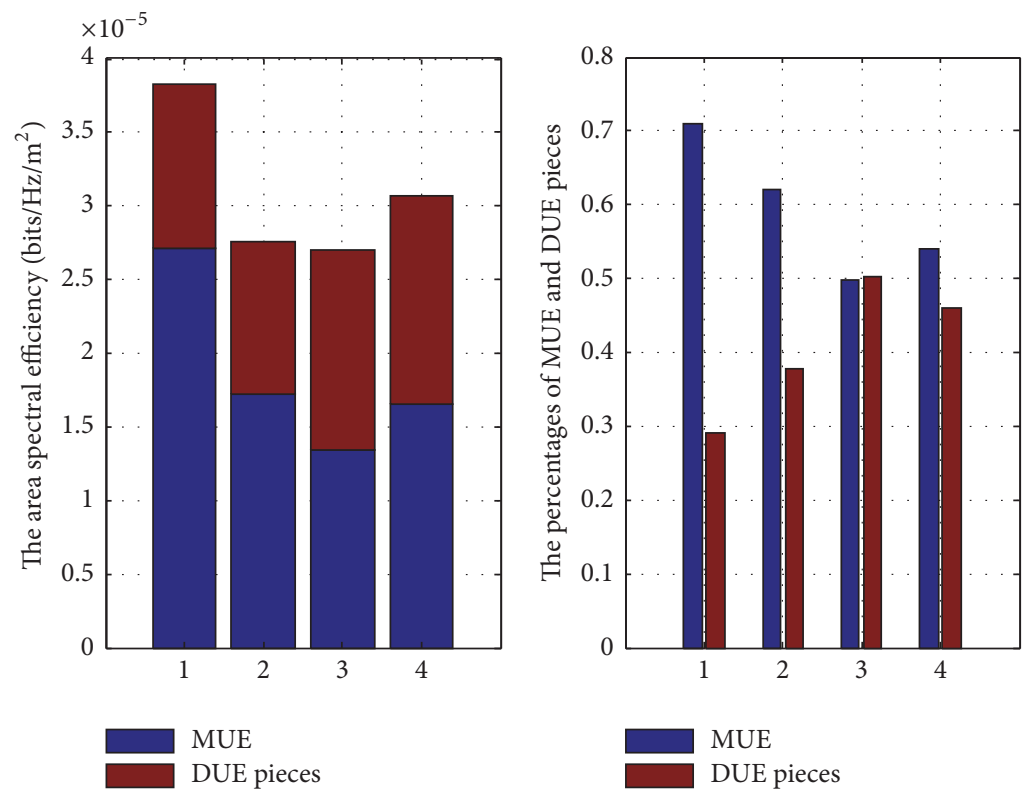

Figure 9: The figure shows $\omega_{1} \Upsilon^{C}(\beta)$ and $\omega_{2} \Upsilon^{D}(\beta)$ with $\omega_{1}=15, \eta=\beta=6 \mathrm{~dB}$, and $\lambda_{\mathrm{DU}}=3 \times 10^{-5}$, respectively.

\section{Conclusions}

The D2D cellular network is an efficient way to carry out mobile crowdsourcing. For D2D underlaid cellular networks, the channel access and power control issue is investigated. In order to limit the cross-tier and intratier interferences, we propose a novel semidistributed network-assisted power and channel access control scheme for DUE pieces. In the proposed semidistributed scheme, with the help of assistant information from the BS, each D2D pair decides whether to access the subchannel and adjusts the transmit power independently. The BS plays a role in offering assistant information, which can maximize the total area spectral efficiency on the condition of guaranteeing the performance of the cellular link. Moreover, assistant information from the BS is statistical, and the optimal access threshold has the closed-form expression. The proposed scheme has the low computational burden and low overhead to broadcast assistant information, which is quite suitable to the practical systems. 


\section{Appendix}

\section{A. The proof of Lemma 3}

By setting $P_{u_{t_{n}}}=P_{u, \max }$, we have

$$
\begin{aligned}
& \Upsilon^{D}(\beta) \leq \lambda_{\mathrm{DU}} P S_{u_{n}} \\
& \cdot \exp \left(-\frac{\pi \lambda_{\mathrm{DU}} P S_{u_{n}} \beta^{2 / \alpha}}{\sin c(2 / \alpha)} \frac{\mathbb{E}\left[P_{u_{t_{n}}}^{2 / \alpha}\right]}{P_{u, \max }^{2 / \alpha}} d_{u_{t_{n}}, u_{r_{n}}}^{2}\right) \\
& \cdot \frac{\log _{2}(1+\beta)}{1+\left(\beta\left(P_{s_{0}} / P_{u, \max }\right)\right)^{2 / \alpha}\left((45 \pi)^{2} d_{u_{t_{n}}, u_{r_{n}}}^{2} /(128 R)^{2}\right)} .
\end{aligned}
$$

Plugging (15) into (A.1), we have (25).

\section{B. The proof of Theorem 4}

The derivative of $f(x)$ with respect to $x$ is written as

$$
\frac{\partial f(x)}{\partial x}=-a \omega_{1} e^{-a x}+\omega_{2} e^{-b x}(1-b x) .
$$

From the first-order optimality condition, that is, $\partial f(x) / \partial x=$ 0 , we have

$$
1-b x=\frac{a \varepsilon_{1}}{\varepsilon_{2}} e^{(b-a) x} .
$$

Denoting that $p=a \varepsilon_{1} / \varepsilon_{2}$ and $q=a-b,(\mathrm{~B} .2)$ is rewritten as

$$
1-b x=p e^{-q x} \text {. }
$$

Then, a closed-form solution of $x$ which satisfies the firstorder optimality condition is derived as

$$
\widehat{x}=\frac{1}{b}+\frac{1}{q} \omega\left(-\frac{p q e^{-q / b}}{b}\right) .
$$

Considering the constraint, that is, $0<x<1$, we have Theorem 4.

\section{Conflicts of Interest}

The authors declare that they have no conflicts of interest.

\section{Acknowledgments}

The research was supported in part by the National Natural Science Foundation of China (Grant nos. 61601482 and 61502518).

\section{References}

[1] Z. Ning, X. Hu, Z. Chen et al., "A cooperative quality-aware service access system for social internet of vehicles," IEEE Internet of Things Journal, pp. 1-1.

[2] Y. Han, T. Luo, D. Li, and H. Wu, "Competition-based participant recruitment for delay-sensitive crowdsourcing applications in D2D networks," IEEE Transactions on Mobile Computing, vol. 15, no. 12, pp. 2987-2999, 2016.
[3] M. Tehrani, M. Uysal, and H. Yanikomeroglu, "Device-todevice communication in 5G cellular networks: challenges, solutions, and future directions," IEEE Communications Magazine, vol. 52, no. 5, pp. 86-92, 2014.

[4] X. Hu, J. Deng, J. Zhao et al. et al., "SAfeDJ: a crowd-cloud codesign approach to situation-aware music delivery for drivers," ACM Transactions on Multimedia Computing, Communications, and Applications (TOMM), vol. 12, no. 1s, article 21, 2015.

[5] X. Hu, T. H. S. Chu, H. C. B. Chan, and V. C. M. Leung, "Vita: a crowdsensing-oriented mobile cyber-physical system," IEEE Transactions on Emerging Topics in Computing, vol. 1, no. 1, pp. 148-165, 2013.

[6] X. Hu, T. H. S. Chu, V. C. M. Leung, E. C.-H. Ngai, P. Kruchten, and H. C. B. Chan, "A survey on mobile social networks: applications, platforms, system architectures, and future research directions," IEEE Communications Surveys \& Tutorials, vol. 17, no. 3, pp. 1557-1581, 2015.

[7] L. Lei, Y. Kuang, X. Shen, C. Lin, and Z. Zhong, "Resource control in network assisted device-to-device communications: solutions and challenges," IEEE Communications Magazine, vol. 52, no. 6, pp. 108-117, 2014.

[8] C. Yu, K. Doppler, C. B. Ribeiro, and O. Tirkkonen, "Resource sharing optimization for device-to-device communication underlaying cellular networks," IEEE Transactions on Wireless Communications, vol. 10, no. 8, pp. 2752-2763, 2011.

[9] J. Gu, S. J. Bae, B.-G. Choi, and M. Y. Chung, "Dynamic power control mechanism for interference coordination of deviceto-device communication in cellular networks," in Proceedings of the 3rd International Conference on Ubiquitous and Future Networks (ICUFN '11), pp. 71-75, June 2011.

[10] N. Y. Lee, X. Q. Lin, J. G. Andrews, and R. W. Heath, "Power control for D2D underlaid cellular networks: modeling, algorithms, and analysis," IEEE Journal on Selected Areas in Communications, vol. 33, no. 1, pp. 1-13, 2015.

[11] S.-H. Yang, L.-C. Wang, J.-H. Huang, and A.-H. Tsai, "Networkassisted device-decided channel selection and power control for multi-pair device-to-device (D2D) communications in heterogeneous networks," in Proceedings of the 2014 IEEE Wireless Communications and Networking Conference, WCNC 2014, pp. 1356-1361, Istanbul, Turkey, April 2014.

[12] P. Sun, K. G. Shin, H. Zhang, and L. He, “Transmit power control for $\mathrm{d} 2 \mathrm{~d}$-underlaid cellular networks based on statistical features," IEEE Transactions on Vehicular Technology, vol. 66, no. 5, pp. 4110-4119, 2017.

[13] D. Moltchanov, "Distance distributions in random networks," Ad Hoc Networks, vol. 10, no. 6, pp. 1146-1166, 2012.

[14] L. Zhou, X. Hu, E. C.-H. Ngai et al., "A dynamic graph-based scheduling and interference coordination approach in heterogeneous cellular networks," IEEE Transactions on Vehicular Technology, vol. 65, no. 5, pp. 3735-3748, 2016.

[15] X. Lin, J. G. Andrews, and A. Ghosh, "Spectrum sharing for device-to-device communication in cellular networks," IEEE Transactions on Wireless Communications, vol. 13, no. 12, pp. 6727-6740, 2014.

[16] D. Stoyan, W. Kendall, and J. Mecke, Stochastic Geometry and Its Applications, Wiley, New York, NY, USA, 1995.

[17] A. Behnad, A. M. Rabiei, and N. C. Beaulieu, "Performance analysis of opportunistic relaying in a poisson field of amplifyand-forward relays," IEEE Transactions on Communications, vol. 61, no. 1, pp. 97-107, 2013.

[18] Z. Chen and M. Kountouris, "Distributed SIR-aware opportunistic access control for D2D underlaid cellular networks," 
in Proceedings of the 2014 IEEE Global Communications Conference, GLOBECOM 2014, pp. 1540-1545, Austin, TX, USA, December 2014.

[19] M. Ehrgott, Multicriteria Optimization, Springer, New York, NY, USA, 2005 


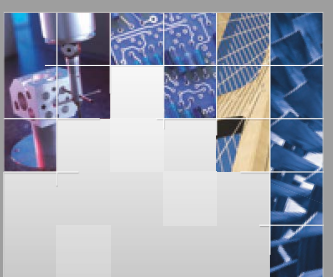

\section{Enfincering}
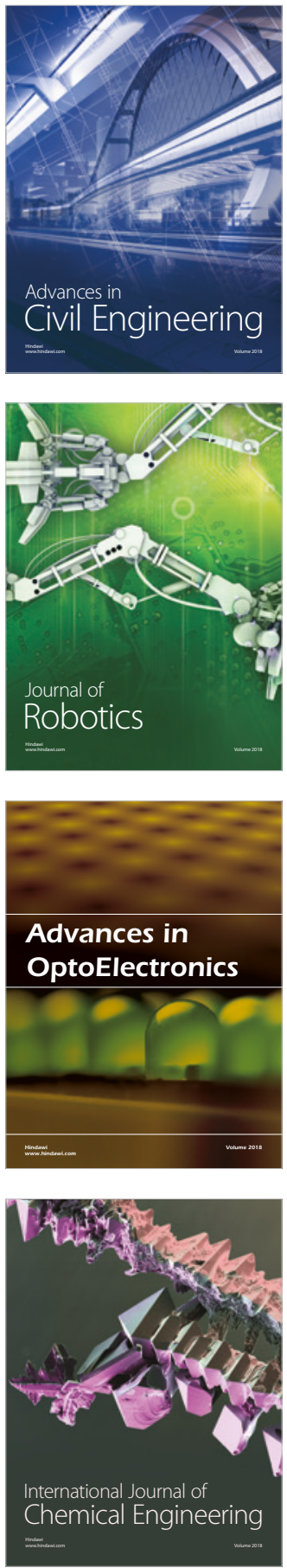

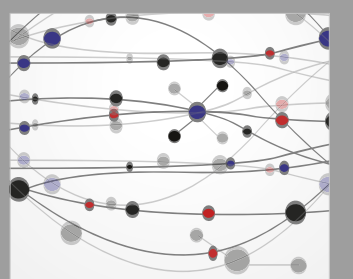

\section{Rotating \\ Machinery}

The Scientific World Journal

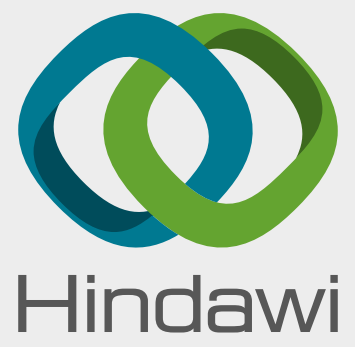

Submit your manuscripts at

www.hindawi.com
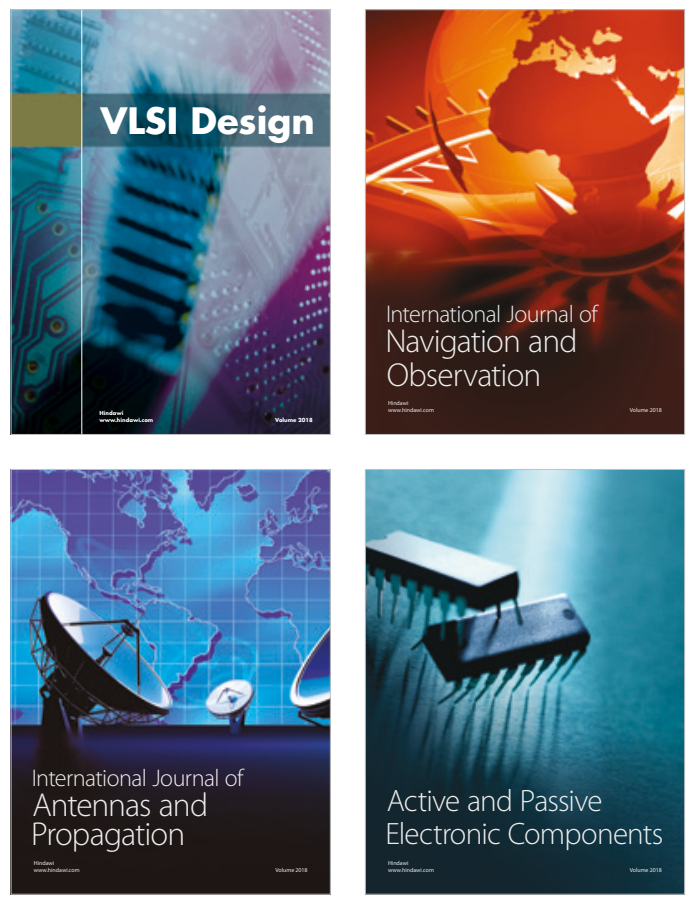
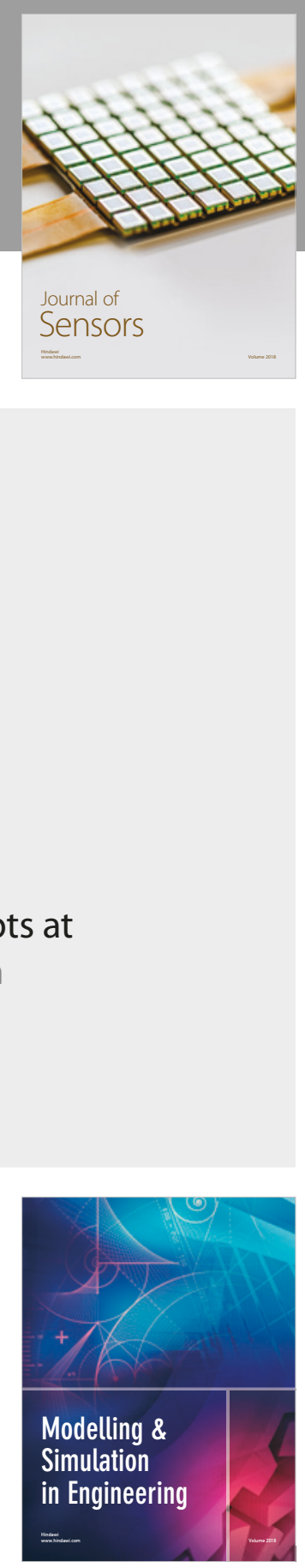

\section{Advances \\ Multimedia}
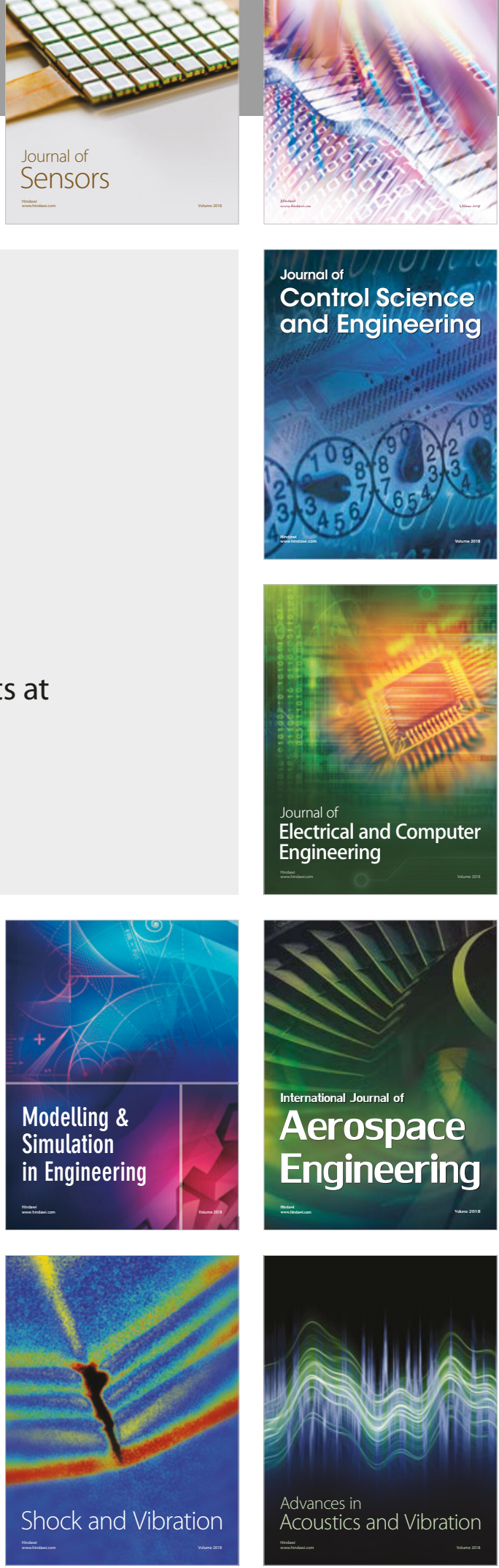